\title{
Influence of a One-Time Web-Based Provider Intervention on Patient-Reported Outcomes After the Well-Child Visit: A Feasibility Study
}

Chad M. Coleman

Gwen L. Alexander

Charles Barone

Andrew S. Bossick

Zeinab Kassem

Mei Lu

Yueren Zhou

Andrea E. Cassidy-Bushrow

Follow this and additional works at: https://aah.org/jpcrr

Part of the Epidemiology Commons, Medical Education Commons, and the Pediatrics Commons

\section{Recommended Citation}

Coleman CM, Alexander GL, Barone C, Bossick AS, Kassem Z, Lu M, Zhou Y, Cassidy-Bushrow AE. Influence of a one-time web-based provider intervention on patient-reported outcomes after the well-child visit: a feasibility study. J Patient Cent Res Rev. 2021;8:48-57. doi: 10.17294/2330-0698.1775

Published quarterly by Midwest-based health system Advocate Aurora Health and indexed in PubMed Central, the Journal of Patient-Centered Research and Reviews (JPCRR) is an open access, peer-reviewed medical journal focused on disseminating scholarly works devoted to improving patient-centered care practices, health outcomes, and the patient experience. 


\title{
Influence of a One-Time Web-Based Provider Intervention on Patient-Reported Outcomes After the Well-Child Visit: A Feasibility Study
}

\author{
Chad M. Coleman, MPH, ${ }^{1}$ Gwen L. Alexander, PhD, ${ }^{1}$ Charles Barone, MD, ${ }^{2}$ Andrew S. Bossick, MPH, ${ }^{1}$ \\ Zeinab Kassem, MD, ${ }^{1}$ Mei Lu, PhD, ${ }^{1}$ Yueren Zhou, MS, ${ }^{1}$ Andrea E. Cassidy-Bushrow, PhD ${ }^{1}$ \\ ${ }^{1}$ Patient-Engaged Research Center, Department of Public Health Sciences, Henry Ford Health System, Detroit, MI; \\ ${ }^{2}$ Department of Pediatric Administration, Henry Ford Health System, Detroit, MI
}

\begin{abstract}
Purpose Patient-centered care promotes positive health outcomes in pediatrics. We created a provider-focused intervention and implemented it in a pragmatic clustered randomized controlled trial to improve healthrelated quality of life (HRQOL) among pediatric patients.
\end{abstract}

Methods A one-time (1-1.5-hour) webinar focusing on patient-centered care and motivational interviewing, using obesity screening as an example, was developed. Pediatric providers were recruited and randomized to either intervention (webinar) or control (usual care) arms. All well-child visits to these providers for a period of up to 5 months following webinar completion (or study enrollment for controls) were identified, and these family/patients were invited to complete a survey to assess HRQOL postvisit. Reported outcomes were compared between intervention and control participants using clustered $t$-tests, chi-squared tests and multiple linear regression models.

Results We recruited 20 providers (10 intervention, 10 control) to the study; 469 parents/guardians and 235 eligible children seeing these providers completed the postvisit survey. Parents/guardians of 8-12-year-old children in the intervention group reported higher school functioning compared to controls (83.5 vs 75.8; $\mathrm{P}=0.023$ ). There were no other differences in children's HRQOL between intervention and control groups.

Conclusions A one-time, web-based provider intervention is feasible to implement in pediatrics. Modest evidence, requiring further study, indicates that instructing providers on patient-centered care in the well-child visit may improve aspects of pediatric HRQOL (ie, school functioning) compared to usual care. However, this was a brief intervention, with multiple outcomes tested and no evaluation of pre- and postintervention provider knowledge, thus additional study is needed. (J Patient Cent Res Rev. 2021;8:48-57.)

Keywords pediatrics; well-child visit; patient-centered; health-related quality of life; provider intervention

\begin{abstract}
$\Lambda$ patient-centered approach to health care incorporates health outcomes meaningful to each individual patient and has been shown to positively influence parent satisfaction in pediatric care. ${ }^{1}$ Similarly encouraging is that patient- and family-centered approaches in the pediatric setting improve health and well-being. For example, Perrin et al conducted a pre/ posttest study to measure the effects of a pediatric provider "toolkit" for preventing and treating childhood obesity, ${ }^{2}$ which included a 1-hour training session to teach pediatric
\end{abstract}

Corresponding author: Andrea E. Cassidy-Bushrow, PhD, Patient-Engaged Research Center, Henry Ford Health System, 1 Ford Place-5C, Detroit, MI 48202

(acassid1@hfhs.org) residents how to deliver patient- and parent-focused weight interventions using the toolkit. The intervention successfully increased parental accuracy of their child's weight status as well as increased healthy dietary choices and physical activity of the participating children in the 3 -month period following the intervention. ${ }^{2}$

Varni and Limbers contend that health-related quality of life (HRQOL) may be "the most important outcome for child health interventions." ${ }^{3}$ There is a growing body of literature on tools for measuring pediatric patientreported outcomes, ${ }^{4-7}$ including measures of HRQOL. Although child self-report is the gold standard, parent/ guardian report is also integral, as the parent/guardian is usually the key health care decision-maker. ${ }^{4,8}$ Few studies have estimated patient-reported outcomes in healthy pediatric patients. Several studies have shown that 
HRQOL, measured using the Pediatric Quality of Life Inventory (PedsQL ${ }^{\mathrm{TM}}$, Mapi Research Trust), is lower in children with obesity compared to those of normal weight, regardless of parent or child report., ${ }^{5,9}$ Indeed, HRQOL of obese pediatric patients has been reported to be as low as that of pediatric patients with cancer. ${ }^{10}$ However, additional studies are needed to understand if improving patient-centered care may improve pediatric HRQOL.

The goal of this pragmatic clustered randomized controlled trial was to examine if a brief, provider-focused course on promoting a patient-centered approach to addressing obesity and obesity-related disease screening and management, versus usual care, would be associated with better pediatric patient and parent/guardian proxy HRQOL, as measured by the PedsQL and the National Institutes of Health's Patient-Reported Outcomes Measurement Information System (PROMIS) Pediatric/ Parent Proxy Profile 25. ${ }^{4}$ Because the intervention specifically included examples of care focused on obesity and related conditions, we also examined a priori if the intervention effect varied by the obesity status of the child at baseline, as we hypothesized the intervention may have a greater impact in visits that included discussions around the child's obesity status.

\section{METHODS}

All study activities were approved by the local institutional review board (\#8287). This study was designed as a clustered randomized controlled trial (NCT02850224), and pediatric providers were the unit of randomization. The providers were randomly assigned to either the intervention (one-time brief informational webinar) or usual care. The unit of measurement was the child patient and their respective parent/guardian (family/patients) of participating providers.

\section{Provider Recruitment and Randomization}

All pediatric providers at Henry Ford Health System (Detroit, MI) conducting well-child visits were eligible to participate. Figure 1 provides a flow diagram of the study activities. Providers were informed of the study at several Department of Pediatrics staff and operations meetings, and emails also were sent to eligible providers inviting them to participate. Interested providers contacted study staff to enroll and provided verbal informed consent to participate in the study by telephone. Using a random digit generator, providers were randomly assigned to intervention or control group (ie, usual care) upon enrollment.

All intervention providers completed the one-time intervention webinar. Control providers were invited to complete the webinar after the patient recruitment

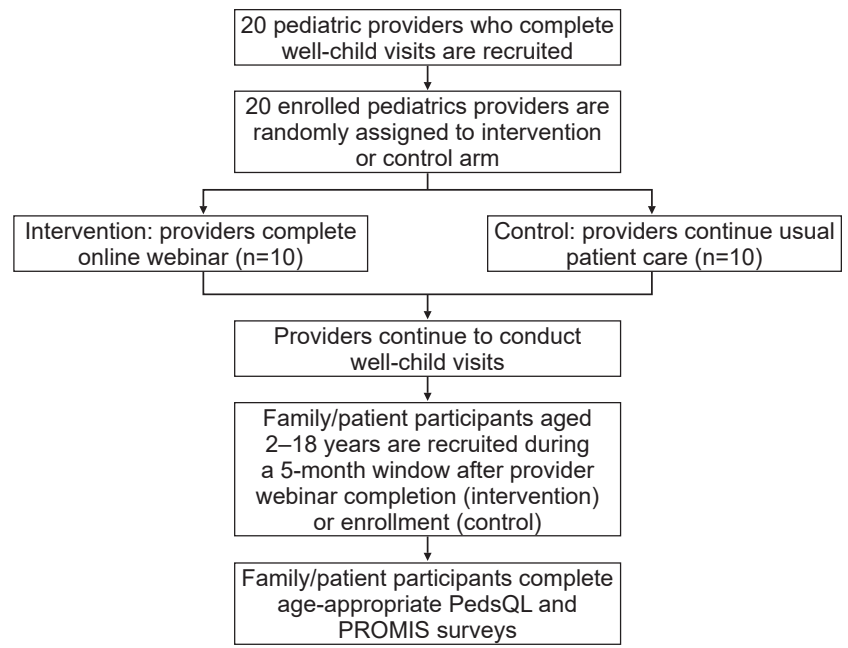

Figure 1. Flow chart of study activities for study providers and family/patient participants. The maximum number of family/patient participants enrolled per provider cluster was 25. PedsQL, Pediatric Quality of Life Inventory; PROMIS, PatientReported Outcomes Measurement Information System Pediatric/Parent Proxy Profile 25.

period ended. From the time of webinar completion (intervention arm) or from the time of notification of the study beginning (control arm), providers were included in the study for a period of 5 months. Provider clusters were limited to 25 family/patient participants, as this sample size adequately accounted for clustering effects in analyses. Once a cluster reached 25 family/patient participants, recruitment stopped for that provider.

\section{Intervention Development}

After conducting focus groups with parents, providers, and pediatric patients (adolescents) to identify knowledge gaps and patient-centered outcomes, ${ }^{11}$ we developed a brief, online webinar-style course for providers aimed at improving patient-centered communication that leads to better patient-centered outcomes in the well-child setting. This intervention was a one-time webinar, which took 1-1.5 hours to complete and could be completed in a single or multiple sittings. The course was divided into 3 modules (less than 20 minutes each) that covered: 1) patient-centered outcomes, including the application of those in pediatric care and techniques for implementation in practice; 2) lessons learned from applying a patientcentered approach in pediatric care, including information from previous focus groups; and 3 ) an introduction to motivational interviewing (MI), including MI coaching techniques that use goal-oriented communication with specific attention to language of change and intrinsic and extrinsic motivation for change after identifying what 
matters most to the patient, and a supporting example of implementing $\mathrm{MI}$ in a pediatric clinical setting.

Given that childhood obesity is a major health issue for children in the area served by Henry Ford Hospital (Detroit, MI), we tailored the course to aid clinicians in conducting a patient-centered approach to pediatric obesity and related disease screening. The course focused on illustrations for incorporating a MI-style person-centered coaching dialogue ${ }^{12,13}$ previously used successfully in behavior change interventions for management of obesity. ${ }^{14}$ The coaching component contained animations illustrating exchanges that incorporated hypothetical scenarios of a pediatric patient and their parent/guardian interacting with the health provider. The scenarios focused on examples of recommendations, questions to uncover ambivalence, and fostering improved patient and parent/guardian understanding around obesity and obesity-related disease screening.

\section{Family/Patient Participant Recruitment}

Data from the electronic medical records at Henry Ford Health System were utilized to identify potential family/ patient participants in the study. For all study providers (intervention and control), we used the date of webinar completion or date of study enrollment, respectively, to identify a 5-month window of eligibility. All patients (and a parent/guardian) of enrolled providers who had a well-child visit within this 5-month window, were 2-18 years of age, were fluent in English, and were able to consent (and, when old enough, assent) to all aspects of participation were eligible for the study unless they were screened as ineligible during the recruitment process, as defined later. International Classification of Diseases, Tenth Revision codes (Z00.00, Z00.01, Z00.110, Z00.11, Z00.121, Z00.129, Z00.5, Z00.8, Z02.1, Z02.2, Z03.3, Z02.4, Z02.5, Z02.6, Z02.71, Z02.79, Z02.81, Z02.82, Z02.83, Z02.89, and Z02.9) and Current Procedural Terminology codes (99381-99385, 99391-99395, and 99461) were used to identify well-child visits.

Manual review of the problems list in the electronic medical record was used to identify potentially ineligible patients. Family/patients were ineligible if the child had a history of a cancer diagnosis, as this diagnosis required more frequent office visits beyond the well-child visit ( $\mathrm{n}=4$ family/patients), or if the child had a diagnosed developmental disorder, which may have resulted in the inability to independently understand and answer survey questions ( $\mathrm{n}=48$ family/patients).

After the well-child visit was completed, family/patients for enrolled providers received an invitation by email if an email address was on file in the medical record or by postal mail. Invited family/patients could join the study by directly accessing the one-time questionnaire either online or on paper by postal mail, if requested. If the family/patient did not respond by 1 week from the invitation mailing, a research assistant followed up with the parent/guardian either by telephone (for those with no email available) or by resending the recruitment email. Family/patient participants were able to complete the survey up to 6 weeks after the well-child visit. All parents/ guardians and children over 10 years old consented/ assented electronically via the online questionnaire system (REDCap, Vanderbilt University) ${ }^{15,16}$ or a physical consent form, depending on if they completed the survey online or by paper.

\section{Family/Patient Survey}

Two validated instruments (PedsQL and PROMIS Pediatric/Parent Proxy Profile) were used to measure the family/patient participant-reported outcomes following the well-child visit. PedsQL is a generic module that is brief, reliable, and valid. ${ }^{5}$ It includes child self-report for those 5-18 years of age and parent proxy report for those 2-18 years of age; parent-report items directly parallel those of the child-report scale. PedsQL measures 4 primary domains: physical, emotional, social, and school functioning. Higher scores indicate higher quality of life in each domain. ${ }^{17}$ The PROMIS Pediatric/Parent Proxy Profile is a collection of short forms assessing anxiety, depressive symptoms, fatigue, pain, physical function/ mobility, and peer relationships. ${ }^{4}$ Forms are available for self-report for children 8 years and older and parent-proxy forms are available for children 5 years and older. Raw scores are converted to a T-score used for standardization. ${ }^{18}$ Higher scores indicate better-than-average outcomes for PROMIS concepts of physical function/mobility and peer relationships, while lower scores indicate betterthan-average outcomes for PROMIS concepts of anxiety, depressive symptoms, fatigue, and pain.

Overall, the burden of questions for parents/guardians was between 60 and 62 total questions and for children old enough to self-report (8-18 years for PROMIS and 5-18 years for PedsQL) was between 23 and 48 total questions. Total time to take the survey was less than 10 minutes for all groups, and parents/guardians and children who completed the survey were mailed a $\$ 5$ cash stipend each upon completion to thank them for their participation.

\section{Obesity and Other Covariates}

Data on age at well-child visit, sex, race, insurance type, height, and weight were obtained from the electronic medical record. Body mass index (BMI) $z$-scores and percentiles were calculated ${ }^{19}$; overweight was defined as BMI between the 85 th and 95th percentile and obesity 
as BMI greater than or equal to the 95th percentile. As some demographic groups were small, we group race into the following 3 categories for analysis: White, Black, and Other (which included American Indian or Alaskan Native, Asian, Native Hawaiian or Pacific Islander, more than one race, other, and unknown). Insurance types were grouped into the categories of public and private.

\section{Statistical Analysis}

Baseline characteristics for patients, including age, sex, race, insurance, and BMI, were compared between the intervention group and the control group using $t$-test for continuous variables and chi-squared test for categorical variables at $\alpha$ of 0.05 , adjusting for the clusters. To assess potential intervention effects, we compared PROMIS and PedsQL T-scores in control and intervention groups by clustered $t$-tests. To investigate if the intervention effect varied by child obesity status, we fit multiple linear regression models, accounting for clustering, with an interaction term between intervention group and obesity status.

\section{RESULTS}

We identified 3544 eligible family/patients with well-child visits from the 20 study providers as potential participants; $1923(54.3 \%)$ were patients of control providers and 1621 (45.7\%) were patients of intervention providers. A total of $483(13.6 \%)$ parents/guardians agreed to participate in the study (with 311 children in eligible age groups agreeing to participate); 238 parents/guardians and 155 children made up the control provider group, and 245 parents/guardians and 156 children made up the intervention provider group. Among those who agreed to participate, 469 (97.1\%) parents/guardians (231 control, 238 intervention) and 235 (75.6\%) children (118 control, 117 intervention) completed the study. This constituted the final analytical set of family/ patient participants in the study.

Table 1 provides descriptive characteristics of the study population. The average age of the children in the study was 8.8 years (standard deviation: 4.7 years), and nearly half were boys (49\%). There were no statistically significant

Table 1. Demographic and Basic Health Characteristics of Pediatric Patients at Time of Well-Child Visit, Overall and by Intervention Status

\begin{tabular}{|c|c|c|c|c|}
\hline Variable & $\begin{array}{l}\text { Overall } \\
(n=469)\end{array}$ & $\begin{array}{l}\text { Control } \\
(n=231)\end{array}$ & $\begin{array}{l}\text { Intervention } \\
(n=238)\end{array}$ & $P$ \\
\hline Age in years & $8.8 \pm 4.7$ & $9.1 \pm 4.8$ & $8.4 \pm 4.7$ & 0.270 \\
\hline \multicolumn{5}{|l|}{ Sex } \\
\hline Female & 238 (51\%) & $111(48 \%)$ & $127(53 \%)$ & \multirow[t]{2}{*}{0.300} \\
\hline Male & $231(49 \%)$ & $120(52 \%)$ & $111(47 \%)$ & \\
\hline \multicolumn{5}{|l|}{ Race } \\
\hline Black & $212(45 \%)$ & $87(38 \%)$ & $125(53 \%)$ & \multirow[t]{3}{*}{0.210} \\
\hline White & 172 (37\%) & $93(40 \%)$ & 79 (33\%) & \\
\hline Other & $85(18 \%)$ & $51(22 \%)$ & $34(14 \%)$ & \\
\hline \multicolumn{5}{|l|}{ Insurance } \\
\hline Private & $299(64 \%)$ & $145(63 \%)$ & $154(64 \%)$ & \multirow[t]{2}{*}{0.826} \\
\hline Public & $170(36 \%)$ & $86(37 \%)$ & $84(35 \%)$ & \\
\hline $\mathrm{BMI}$ in $\mathrm{kg} / \mathrm{m}^{2}$ & $19.8 \pm 5.64$ & $19.9 \pm 5.84$ & $19.7 \pm 5.45$ & 0.740 \\
\hline BMI-for-age $z$-score & $0.5 \pm 1.24$ & $0.5 \pm 1.28$ & $0.6 \pm 1.21$ & 0.480 \\
\hline BMI-for-age percentile & $64.4 \pm 30.1$ & $63.2 \pm 30.4$ & $65.6 \pm 29.8$ & 0.420 \\
\hline \multicolumn{5}{|l|}{ BMI category } \\
\hline Underweight & $22(5 \%)$ & $11(5 \%)$ & $11(5 \%)$ & \multirow[t]{4}{*}{0.980} \\
\hline Normal weight & $277(59 \%)$ & $138(60 \%)$ & $139(58 \%)$ & \\
\hline Overweight & $75(16 \%)$ & $37(16 \%)$ & $38(16 \%)$ & \\
\hline Obese & $95(20 \%)$ & $45(19 \%)$ & $50(21 \%)$ & \\
\hline
\end{tabular}

Sample sizes are based on parent/guardian sample. Data are presented as $n(\%)$ or mean \pm standard deviation. $P$-values compare control and intervention groups.

$B M I$, body mass index. 
differences between intervention and control children with respect to all demographic categories, including race, age, insurance status, or sex, and no differences by BMI, BMI percentile, BMI $z$-score, or BMI category.

Table 2 presents the difference in PROMIS scores between intervention and control groups, by respondent type (parent proxy or child response, when applicable), applicable age category, and question category (anxiety, depressive symptoms, fatigue, peer relationships, physical function mobility, and pain). There was no evidence of any differences in pediatric patients of intervention compared to control group providers in any PROMIS measures $(\mathrm{P}>0.05$ for all).
Table 3 presents PedsQL scores by intervention status, respondent type (parent proxy or child response, when applicable), applicable age category, and question category (physical, emotional, social, and school functioning). There was one difference noted in pediatric patients of intervention compared to control group providers. Parents/guardians of 8-12-year-old children reported significantly higher school functioning among intervention children than control children (mean score of $83.50 \pm 16.65$ vs $75.80 \pm 21.96 ; \mathrm{P}=0.023)$.

Because the intervention webinar used examples that focused on obesity screening, we also examined if there was evidence that obesity acted as an effect modifier.

Table 2. PROMIS Results by Intervention Status for Parent Proxy and Self-Report, by Age Group

\begin{tabular}{|c|c|c|c|c|c|c|}
\hline \multirow[b]{2}{*}{ Construct } & \multicolumn{3}{|c|}{ Self-report } & \multicolumn{3}{|c|}{ Parent proxy } \\
\hline & $\begin{array}{l}\text { Control, } \\
\text { mean (SD) }\end{array}$ & $\begin{array}{l}\text { Intervention, } \\
\text { mean (SD) }\end{array}$ & $P$ & $\begin{array}{l}\text { Control, } \\
\text { mean (SD) }\end{array}$ & $\begin{array}{l}\text { Intervention, } \\
\text { mean (SD) }\end{array}$ & $P$ \\
\hline \multicolumn{7}{|l|}{ Ages 5-7 } \\
\hline $\mathrm{N}$ & & & & 33 & 42 & \\
\hline Anxiety & & & & $44.02(11.10)$ & $43.31(10.05)$ & 0.766 \\
\hline Depressive symptoms & & & & $41.21(6.28)$ & $42.17(8.55)$ & 0.570 \\
\hline Peer relationships & & & & $52.00(10.60)$ & $52.67(9.97)$ & 0.761 \\
\hline Fatigue & & & & $38.33(6.61)$ & $36.76(6.92)$ & 0.306 \\
\hline Physical function mobility & & & & $52.88(5.67)$ & $54.67(4.16)$ & 0.125 \\
\hline \multicolumn{7}{|l|}{ Ages 8-12 } \\
\hline $\mathrm{N}$ & 51 & 44 & & 72 & $70^{\mathrm{a}}$ & \\
\hline Anxiety & $42.69(8.48)$ & $42.63(7.69)$ & 0.974 & $43.35(10.68)$ & $44.03(9.01)$ & 0.677 \\
\hline Depressive symptoms & $43.02(6.80)$ & $43.11(7.10)$ & 0.948 & $43.38(9.00)$ & $43.05(8.67)$ & 0.811 \\
\hline Peer relationships & $51.42(11.68)$ & $51.23(9.26)$ & 0.924 & $49.29(13.32)$ & $51.00(12.12)$ & 0.426 \\
\hline Fatigue & $39.29(5.58)$ & $40.67(8.77)$ & 0.363 & $38.83(7.52)$ & $38.63(7.51)$ & 0.870 \\
\hline Physical function mobility & $55.19(5.33)$ & $55.01(4.97)$ & 0.863 & $53.15(7.55)$ & $52.84(6.19)$ & 0.790 \\
\hline Pain Interference & $44.32(10.06)$ & $45.69(11.34)$ & 0.550 & & & \\
\hline \multicolumn{7}{|l|}{ Ages $13-18$} \\
\hline$N$ & $46^{b}$ & $42^{c}$ & & $68^{\mathrm{d}, \mathrm{e}}$ & 55 & \\
\hline Anxiety & $43.39(11.10)$ & $42.22(8.23)$ & 0.595 & $42.35(9.64)$ & $42.71(9.30)$ & 0.829 \\
\hline Depressive symptoms & $42.99(7.94)$ & $44.57(8.91)$ & 0.389 & $42.40(8.87)$ & $43.58(9.86)$ & 0.473 \\
\hline Peer relationships & $52.03(10.10)$ & $49.41(9.24)$ & 0.205 & $50.03(12.67)$ & $48.20(10.49)$ & 0.388 \\
\hline Fatigue & $42.94(10.25)$ & $42.25(9.27)$ & 0.735 & $41.85(9.45)$ & $42.20(9.90)$ & 0.847 \\
\hline Physical function mobility & $54.56(6.16)$ & $53.26(5.87)$ & 0.313 & $51.98(6.85)$ & $51.71(6.27)$ & 0.814 \\
\hline Pain Interference & $43.51(9.18)$ & $47.11(10.99)$ & 0.081 & & & \\
\hline
\end{tabular}

${ }^{a} \mathrm{~N}=69$ for peer relationships and physical function mobility constructs.

${ }^{b} \mathrm{~N}=45$ for pain interference construct.

${ }^{c} N=40$ for pain interference construct.

${ }^{d} N=67$ for peer relationships and fatigue constructs.

${ }^{e} N=66$ for physical function mobility construct.

PROMIS, Patient-Reported Outcomes Measurement Information System Pediatric/Parent Proxy Profile 25; SD, standard deviation. 
Table 3. PedsQL Results by Intervention Status for Parent Proxy and Self-Report, by Age Group

\begin{tabular}{|c|c|c|c|c|c|c|}
\hline \multirow[b]{2}{*}{ Construct } & \multicolumn{3}{|c|}{ Self-report } & \multicolumn{3}{|c|}{ Parent proxy } \\
\hline & $\begin{array}{c}\text { Control, } \\
\text { mean (SD) }\end{array}$ & $\begin{array}{l}\text { Intervention, } \\
\text { mean (SD) }\end{array}$ & $P$ & $\begin{array}{c}\text { Control, } \\
\text { mean (SD) }\end{array}$ & $\begin{array}{l}\text { Intervention, } \\
\text { mean (SD) }\end{array}$ & $P$ \\
\hline \multicolumn{7}{|l|}{ Ages 2-4 } \\
\hline $\mathrm{N}$ & & & & $58^{a}$ & $70^{\mathrm{b}}$ & \\
\hline Physical functioning & & & & $96.10(9.45)$ & $92.90(14.80)$ & 0.279 \\
\hline Emotional functioning & & & & $91.00(11.91)$ & $91.10(11.39)$ & 0.965 \\
\hline Social functioning & & & & $94.20(7.81)$ & $94.10(8.07)$ & 0.797 \\
\hline School functioning & & & & $90.70(15.65)$ & $91.90(14.43)$ & 0.650 \\
\hline \multicolumn{7}{|l|}{ Ages 5-7 } \\
\hline $\mathrm{N}$ & 24 & 32 & & 33 & 42 & \\
\hline Physical functioning & $97.40(4.88)$ & $94.90(8.47)$ & 0.240 & $87.50(19.99)$ & $89.70(22.58)$ & 0.663 \\
\hline Emotional functioning & $84.20(17.92)$ & $87.80(13.38)$ & 0.377 & $88.20(14.99)$ & $89.50(13.65)$ & 0.668 \\
\hline Social functioning & $89.20(12.48)$ & $90.30(12.04)$ & 0.709 & $90.50(15.88)$ & $87.70(20.81)$ & 0.536 \\
\hline School functioning & 88.80 (15.69) & $85.80(15.51)$ & 0.460 & $87.80(15.29)$ & $87.30(15.47)$ & 0.870 \\
\hline \multicolumn{7}{|l|}{ Ages 8-12 } \\
\hline $\mathrm{N}$ & 51 & 45 & & 72 & 70 & \\
\hline Physical functioning & $92.70(9.67)$ & $90.80(13.49)$ & 0.427 & $82.00(27.18)$ & $86.80(19.29)$ & 0.226 \\
\hline Emotional functioning & $85.50(15.04)$ & $85.80(16.24)$ & 0.930 & $84.00(20.05)$ & $86.70(14.59)$ & 0.354 \\
\hline Social functioning & $89.70(14.40)$ & $87.80(16.36)$ & 0.521 & $84.00(23.72)$ & $87.80(18.25)$ & 0.295 \\
\hline School functioning & $84.80(17.80)$ & $84.00(14.98)$ & 0.812 & $75.80(21.96)$ & $83.50(16.65)$ & 0.023 \\
\hline \multicolumn{7}{|l|}{ Ages 13-18 } \\
\hline $\mathrm{N}$ & 45 & $42^{c}$ & & 68 & $54^{\mathrm{d}, \mathrm{e}}$ & \\
\hline Physical functioning & $91.50(14.47)$ & $91.40(11.67)$ & 0.996 & $85.60(22.93)$ & $83.80(21.63)$ & 0.657 \\
\hline Emotional functioning & $85.40(20.47)$ & $87.30(16.57)$ & 0.639 & $85.70(18.15)$ & $87.00(14.14)$ & 0.686 \\
\hline Social functioning & $91.10(17.15)$ & $88.20(15.60)$ & 0.382 & $85.40(22.85)$ & $86.00(15.54)$ & 0.875 \\
\hline School functioning & $83.80(20.09)$ & $78.30(20.07)$ & 0.202 & $81.70(21.18)$ & $79.00(20.60)$ & 0.482 \\
\hline
\end{tabular}

${ }^{a} \mathrm{~N}=25$ for school functioning construct.

${ }^{b} \mathrm{~N}=38$ for school functioning construct.

${ }^{c} N=41$ for social functioning and school functioning constructs.

${ }^{d} N=52$ for emotional functioning construct.

${ }^{e} N=53$ for social functioning and school functioning constructs.

PedsQL, Pediatric Quality of Life Inventory; SD, standard deviation.

Depending on child obesity status, there were differences in the pediatric patients of intervention compared to control providers for the PROMIS peer relationship construct reported by parents/guardians of children 13-18 years old $(\mathrm{P}=0.014)$. Specifically, while parents/guardians of nonobese children reported better peer relationships in the intervention compared to control group (mean peer relationships score of $53.50 \pm 8.46$ for intervention vs 50.13 \pm 12.81 for control), among parents/guardians of children with obesity, those in the control group reported higher peer relationship scores compared to those in the intervention group (mean peer relationships score of $42.70 \pm 9.63$ for intervention vs $49.90 \pm 12.71$ for control) (Figure 2).
Also per child obesity status, there were differences in the pediatric patients of intervention compared to control providers in self-reported school functioning (PedsQL) in children 5-7 years old $(\mathrm{P}=0.034)$. Among children with obesity, mean school functioning scores were $90.00 \pm$ 14.14 and $80.00 \pm 18.52$ in the intervention and control groups, respectively, whereas among children without obesity, mean school functioning scores were $84.13 \pm$ 16.00 and $93.13 \pm 12.50$ for intervention and control, respectively (Figure 3 ). There was no other evidence of differences in pediatric patients of intervention and control providers by child obesity status on any other PROMIS or PedsQL measure ( $\mathrm{P} \geq 0.099$ for all interactions). 

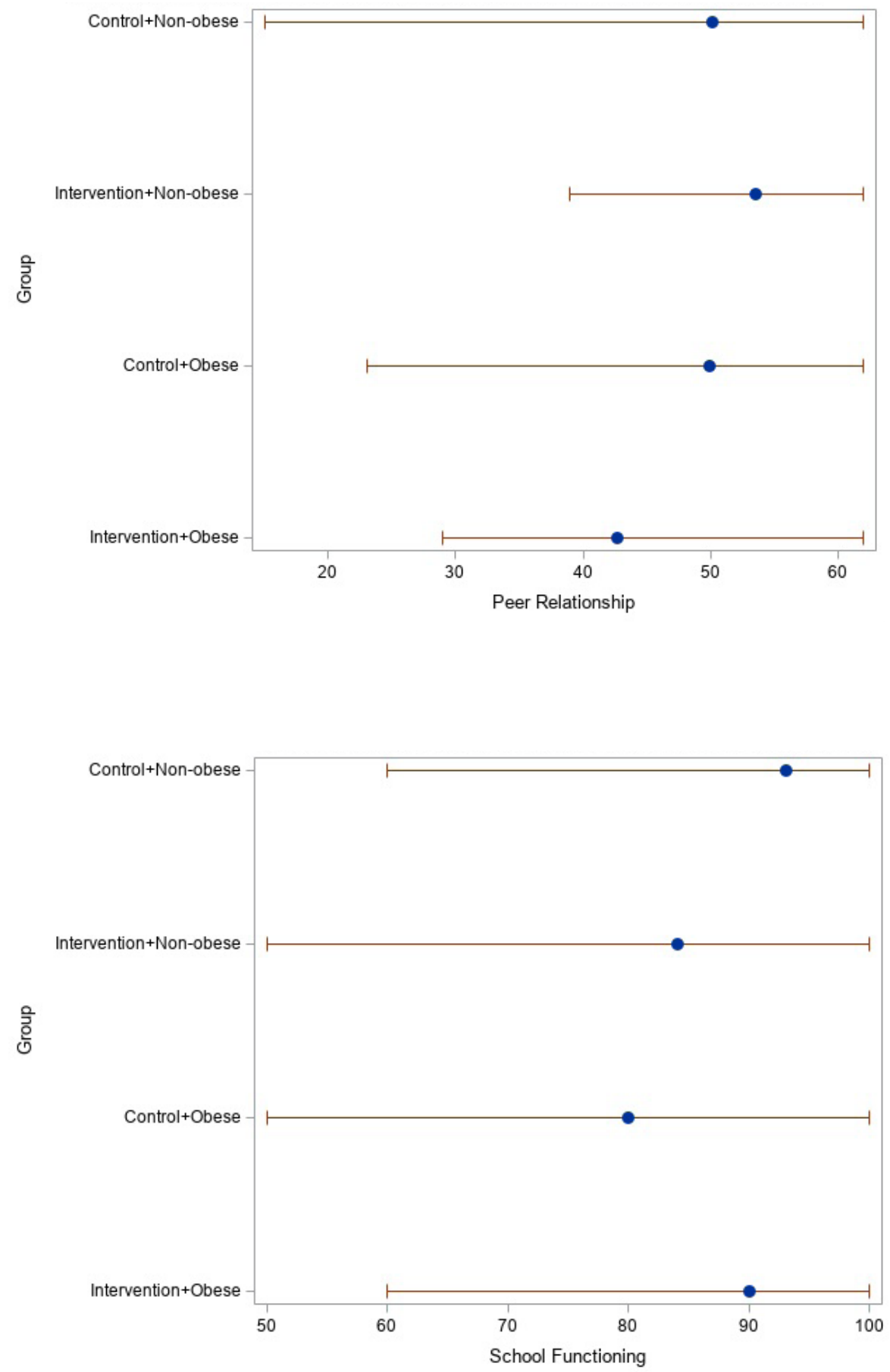

Figure 2. Mean parent-reported peer relationship scores, with 95\% confidence intervals, for 13-18-year-old children by intervention and obesity status.
Figure 3. Mean school functioning scores, with 95\% confidence intervals, for 5-7-year-old children (selfreported) by intervention and obesity status.

\section{DISCUSSION}

Our study demonstrates it is feasible to recruit pediatric providers to an intervention consisting of a one-time webinar focusing on patient-centered care. Our findings introduce supportive data that, compared to children receiving usual care, children seeing a provider who completed the webinar had a modestly higher score in one domain of HRQOL, specifically, parent/guardianreported school functioning in children 8-12 years old.
We also found some evidence that obesity modified the impact of the intervention on school functioning in children 5-7 years of age and on peer relationships in children 13-18 years of age, as compared to the control arm.

Parents of 8-12-year-old children in the intervention group reported better school functioning scores than those in the control group. Examples of patient-centered care offered by the intervention involved asking the 
pediatric patient what mattered to them (eg, topics related to fitting in at school such as "What things are you really happy about related to school?"). It is possible that the intervention providers, when probing for potential outcomes of interest to their patient, were able to provide tailored feedback related to school situations. Similar differences in school functioning scores have been reported in other pediatric intervention studies. In the Go4it trial of obese adolescents assigned to either cognitive behavior therapy sessions or regular care with the addition of a dietician, school functioning scores were significantly higher in the intervention (mean score: $76.6 \pm 16.2$ ) compared to control group (mean score: $71.7 \pm 14.4$ ) at 18 months postintervention. ${ }^{20}$ The authors attributed the improvement in school functioning scores to the cognitive behavior therapy aspects of the intervention, which may have improved adolescents' ability to react to difficult situations and/or teasing. Additional study of the potential mechanisms by which interventions with pediatric providers may improve a patient's school functioning are needed.

The intervention deployed in our study utilized MI principles. MI has been used as a brief intervention, important in settings that could have compromised communication effectiveness due to appointment time constraints. ${ }^{21}$ MI approaches to encourage behavior changes work effectively as a standalone intervention for pediatric providers to incorporate into care practices. ${ }^{22}$ However, there is limited training in MI for pediatric providers. In a meta-analysis of MI and pediatric behavior change, Gayes and Steele found that MI effectiveness was determined by provider background. ${ }^{23}$ Incorporating MI training in pediatric residency, fellowship programs, and continuing medical education may improve health care delivery and patient-centered outcomes by introducing the concept early in clinical training and allowing providers to feel comfortable with and experience the value of the skill.

Our intervention focused on examples of pediatric care of obesity and obesity-related disease. There were statistically significant differences in PedsQL and PROMIS scores for pediatric patients of intervention versus control providers on peer relationships in children 13-18 years of age and school functioning in children 5-7 years of age; however, the direction of impact of obesity status on the intervention varied by the outcome. The PROMIS tool does not have a specific school functioning construct, making direct comparison to the PedsQL difficult. While both tools have peer relationship constructs, the topics and wording of the questions differ (for example, PROMIS measures focus more on friendships and being able to count on friends, while
PedsQL measures focus on teasing and being able to keep up with peers). MI has successfully improved obesity outcomes among children ${ }^{24}$; however, less is known on the effect of MI on HRQOL in children with obesity. Our preliminary findings support future intervention studies specifically focused on addressing the known poorer HRQOL of children with obesity. $5,7,9$

We found differences in HRQOL of pediatric patients of intervention versus control providers within specific age groups. In a review of MI in pediatric populations by Erickson et al, while it was found that MI is successful in adolescent populations to improve self-efficacy and initiate health-related change, there is limited understanding of the effects of MI on younger children. ${ }^{25}$ Specifically, younger children are less likely to generate long-term goals from MI-style coaching. Future research is needed to better understand the impact of MI and provider intervention on HRQOL outcomes for younger children, and such provider-focused interventions should tailor their trainings to consider MI by different age groups. Additionally, tailoring interventions that build on current age-specific pediatric well-child visit priorities may improve patient-reported outcomes without adding to the burden on providers and patients during these visits. Per the American Academy of Pediatrics and Bright Futures periodicity schedule, early childhood care revolves around development, growth, and vaccination schedules, ${ }^{26}$ middle childhood visits (age: $5-10$ years) emphasize the need to evaluate development, success in school, and foundational health practices like teethbrushing and safety, ${ }^{27}$ and during years of adolescence (11-21), providers focus on maturation and health behaviors for the future self. This exemplifies the potential need to tailor MI interventions toward the recommended goals by age group to elicit change for patient-centered outcomes. Future studies that focus on these specific age groups, rather than the broad age range of the study presented herein, may yield stronger findings.

There are a number of strengths to our study. We utilized a pragmatic clustered randomized controlled trial design, which reduces the chance of introducing selection bias and issues with unmeasured confounding. The use of standardized questionnaires to evaluate HRQOL postintervention allows for better understanding of patient-centered outcomes. There is a paucity of randomized controlled trial studies evaluating pediatric patient-reported outcomes ${ }^{28}$; our clinical trial utilizing two established tools to measure pediatric HRQOL shows the feasibility of incorporating these measures in future studies. The one-time education intervention was easy to complete and could be completed online. 


\section{Limitations}

We did not conduct a baseline pretest with providers to assess their understanding of MI and patient-centered principles before the intervention. We did not evaluate provider uptake of the intervention principles; MI is somewhat difficult to master, ${ }^{13}$ and limited competence can be a barrier to implementing the training into practice. Thus, further practice and evaluation of communication content and delivery is needed for reporting full competence of the patient-centered approach to care presented in this intervention. HRQOL outcomes were measured shortly after the intervention, therefore we have limited understanding of the impact of MI at expanded time points.

Only a small number of eligible children participated in the study; while some providers reached the maximum number of patients recruited from their clinic, this largely, but not solely, reflects their patient volume. It is possible that we are still subject to selection bias comparing families who did and did not participate in the study. Providers in both the intervention and control groups were made aware of the purpose of the study, and some providers in both groups practiced out of the same clinic. Thus, there is the potential for contamination of study results if intervention providers shared webinar information with control providers. In analyses examining the intervention effect within obesity group, some of the differences that were found to be significantly different had relatively small effect sizes (ie, for peer relationships in children 13-18 years old). Previous studies suggest a 4.4- to 4.5unit difference in PedsQL total score may relate to a clinically meaningful difference, ${ }^{6,29}$ therefore some of the statistically significant differences we detected may not be clinically meaningful.

Finally, we had a large number of subgroups and statistical tests. Given that little is known on this topic and that this study was largely designed to show feasibility and to generate hypotheses for future studies,,$^{30}$ we chose not to account for multiple hypothesis testing issues. Thus, our findings could be simply due to chance.

\section{CONCLUSIONS}

The research findings presented herein show that it is feasible to recruit pediatric providers to participate in an online webinar-style intervention that focused on a patient-centered approach to pediatric care. Preliminary data suggest that preadolescent patients of providers completing the webinar had higher health-related quality of life in the domain of school functioning. Differences in pediatric patient HRQOL between those seen by intervention- or control-group providers may vary by child age and obesity status. Incorporating patient-centered care in pediatric training programs and continuing medical education may improve health care communication. However, this hypothesis requires additional study.

\section{Patient-Friendly Recap}

- The authors developed a brief, one-time webinar that focused on how to provide patient-centered care in pediatric practice.

- A randomized control trial designed to test the webinar successfully recruited 20 providers to participate, establishing feasibility.

- Few differences in health-related quality of life, as reported by parents or children, were found between those cared for by providers exposed to the webinar versus other providers. One exception was better school functioning scores in 8-12-yearold children seen by the former.

- Further study on the patient-centered care and motivational interviewing approach in pediatric wellchild visits is warranted.

\section{Author Contributions}

Study design: Alexander, Barone, Bossick, Lu, Cassidy-Bushrow. Data acquisition or analysis: Coleman, Bossick, Kassem, Lu, Zhou, Cassidy-Bushrow. Manuscript drafting: Coleman, Zhou, CassidyBushrow. Critical revision: all authors.

\section{Conflicts of Interest}

None.

\section{Funding Sources}

This research was funded by the Agency for Healthcare Research and Quality (R24HS022417). The study sponsors did not participate in data collection, analysis, interpretation, or writing of this manuscript.

\section{References}

1. October TW, Hinds PS, Wang J, Dizon ZB, Cheng YI, Roter DL. Parent satisfaction with communication is associated with physician's patient-centered communication patterns during family conferences. Pediatr Crit Care Med. 2016;17:490-7. CrossRef

2. Perrin EM, Jacobson Vann JC, Benjamin JT, Skinner AC, Wegner S, Ammerman AS. Use of a pediatrician toolkit to address parental perception of children's weight status, nutrition, and activity behaviors. Acad Pediatr. 2010;10:274-81. CrossRef

3. Varni JW, Limbers CA. The pediatric quality of life inventory: measuring pediatric health-related quality of life from the perspective of children and their parents. Pediatr Clin North Am. 2009;56:843-63. CrossRef

4. Irwin DE, Gross HE, Stucky BD, et al. Development of six PROMIS pediatrics proxy-report item banks. Health Qual Life Outcomes. 2012;10:22. CrossRef 
5. Varni JW, Seid M, Rode CA. The PedsQL: measurement model for the pediatric quality of life inventory. Med Care. 1999;37:126-39. CrossRef

6. Varni JW, Burwinkle TM, Seid M. The PedsQL as a pediatric patient-reported outcome: reliability and validity of the PedsQL Measurement Model in 25,000 children. Expert Rev Pharmacoecon Outcomes Res. 2005;5:705-19. CrossRef

7. Varni JW, Limbers CA, Burwinkle TM. Impaired healthrelated quality of life in children and adolescents with chronic conditions: a comparative analysis of 10 disease clusters and 33 disease categories/severities utilizing the PedsQL 4.0 Generic Core Scales. Health Qual Life Outcomes. 2007;5:43. CrossRef

8. Janicke DM, Finney JW, Riley AW. Children's health care use: a prospective investigation of factors related to care-seeking. Med Care. 2001;39:990-1001. CrossRef

9. Kuhl ES, Rausch JR, Varni JW, Stark LJ. Impaired healthrelated quality of life in preschoolers with obesity. $J$ Pediatr Psychol. 2012;37:1148-56. CrossRef

10. Schwimmer JB, Burwinkle TM, Varni JW. Health-related quality of life of severely obese children and adolescents. JAMA. 2003;289:1813-9. CrossRef

11. Bossick AS, Barone C, Alexander GL, Olden H, Troy T, Cassidy-Bushrow AE. Teen, parent, and clinician expectations about obesity and related conditions during the annual wellchild visit. J Patient Cent Res Rev. 2017;4:114-24. CrossRef

12. Miller WR, Rollnick S. Motivational Interviewing: Preparing People for Change, Second Edition. Guilford Press; 2002.

13. Miller WR, Rollnick S. Ten things that motivational interviewing is not. Behav Cogn Psychother. 2009;37:129-40. CrossRef

14. Resnicow K, Davis R, Rollnick S. Motivational interviewing for pediatric obesity: conceptual issues and evidence review. $J$ Am Diet Assoc. 2006;106:2024-33. CrossRef

15. Harris PA, Taylor R, Minor BL, et al. The REDCap consortium: building an international community of software platform partners. J Biomed Inform. 2019;95:103208. CrossRef

16. Harris PA, Taylor R, Thielke R, Payne J, Gonzalez N, Conde JG. Research electronic data capture (REDCap) - a metadatadriven methodology and workflow process for providing translational research informatics support. J Biomed Inform. 2009;42:377-81. CrossRef

17. Varni JW. Scaling and Scoring of the Pediatric Quality of Life Inventory $^{\mathrm{TM}}$ (PedsQLTM), Version 17. Mapi Research Trust; 2017.

18. HealthMeasures. PROMIS pediatric and parent proxy profile instruments. Posted January 15, 2020; accessed March 30, 2020. http://www.healthmeasures.net/images/PROMIS/manuals/ PROMIS_Pediatric and_Proxy_Profile_Scoring_Manual.pdf
19. Kuczmarski RJ, Ogden CL, Guo SS, et al. 2000 CDC growth charts for the United States: methods and development. Vital Health Stat 11. 2002;(246):1-190.

20. Hofsteenge GH, Weijs PJ, Delemarre-van de Waal HA, de Wit M, Chinapaw MJ. Effect of the Go4it multidisciplinary group treatment for obese adolescents on health related quality of life: a randomised controlled trial. BMC Public Health. 2013;13:939. CrossRef

21. Rubak S, Sandbaek A, Lauritzen T, Christensen B. Motivational interviewing: a systematic review and meta-analysis. $\mathrm{Br} \mathrm{J} \mathrm{Gen}$ Pract. 2005;55:305-12.

22. Suarez M, Mullins S. Motivational interviewing and pediatric health behavior interventions. J Dev Behav Pediatr. 2008;29:417-28. CrossRef

23. Gayes LA, Steele RG. A meta-analysis of motivational interviewing interventions for pediatric health behavior change. J Consult Clin Psychol. 2014;82:521-35. CrossRef

24. Christie D, Channon S. The potential for motivational interviewing to improve outcomes in the management of diabetes and obesity in paediatric and adult populations: a clinical review. Diabetes Obes Metab. 2014;16:381-7. CrossRef

25. Erickson SJ, Gerstle M, Feldstein SW. Brief interventions and motivational interviewing with children, adolescents, and their parents in pediatric health care settings: a review. Arch Pediatr Adolesc Med. 2005;159:1173-80. CrossRef

26. Bright Futures/American Academy of Pediatrics. Recommendations for preventive pediatric health care. Posted March 2020; accessed March 30, 2020. https://downloads.aap. org/AAP/PDF/periodicity_schedule.pdf

27. Hagan JF, Shaw JS, Duncan PM (eds). Bright Futures: Guidelines for Health Supervision of Infants, Children, and Adolescents, Third Edition. American Academy of Pediatrics; 2008.

28. Neame MT, Chacko J, Kirkham JJ, Sinha IP, Hawcutt DB. Patient-focused outcomes are infrequently reported in pediatric health information technology trials: a systematic review. J Clin Epidemiol. 2020;119:117-25. CrossRef

29. Varni JW, Burwinkle TM, Seid M, Skarr D. The PedsQL 4.0 as a pediatric population health measure: feasibility, reliability, and validity. Ambul Pediatr. 2003;3:329-41. CrossRef

30. Roback PJ, Askins RA. Judicious use of multiple hypothesis tests. Conserv Biol. 2005;19:261-7. CrossRef

(C) 2021 Advocate Aurora Health, Inc. 